\title{
A Note on Solitary Wave Solutions of the Nonlinear Generalized Camassa-Holm Equation
}

\author{
Lei Zhang and Xing Tao Wang \\ Department of Mathematics, Harbin Institute of Technology, Harbin 150001, China \\ Correspondence should be addressed to Xing Tao Wang; xingtao@hit.edu.cn
}

Received 24 September 2012; Revised 6 January 2013; Accepted 10 January 2013

Academic Editor: Baruch Cahlon

Copyright (C) 2013 L. Zhang and X. T. Wang. This is an open access article distributed under the Creative Commons Attribution License, which permits unrestricted use, distribution, and reproduction in any medium, provided the original work is properly cited.

We give a simple method for applying ordinary differential equation to solve the nonlinear generalized Camassa-Holm equation $u_{t}+2 k u_{x}-u_{x x t}+a u^{m} u_{x}-2 u_{x} u_{x x}+u u_{x x x}=0$. Furthermore we give a new ansätz. In the cases where $m=1,2,3$, the numerical simulations demonstrate the results.

\section{Introduction}

A new dispersive shallow water equation

$$
u_{t}+\kappa u_{x}-u_{x x t}+3 u u_{x}=2 u_{x} u_{x x}+u u_{x x x}
$$

known as the Camassa-Holm equation has been derived by Camassa and Holm [1]. They showed that the CamassaHolm equation has peaked wave solitary solutions which have the first derivative discontinuity at the wave peak called "peakons." In [2], the integral bifurcation method was used to study a nonlinearly dispersive wave equation of Camassa-Holm equation type. In [3], attractor for a coupled nonhomogeneous Camassa-Holm equation with periodic boundary condition was investigated by means of several inequalities. In [4], sufficient conditions for the modified twocomponent Camassa-Holm system were established. In [5], a class of nonlinear fourth order analogue of a generalized Camassa-Holm equation was studied by using sine-cosine method. In [6], He et al. studied a generalized Camassa-Holm equation. In [7], Wazwaz established solitary wave solutions to the modified forms of Degasperis-Procesi and CamassaHolm equations. In [8], a family of Camassa-Holm equations with distinct parameters was investigated. Also many aspects of the problems were studied by researchers [9-16]. Authors in $[4,17]$ presented periodic wave solutions and traveling wave solutions for some equations. In [18], Khuri investigated the periodic wave and peaked solitary wave solutions of the nonlinear generalized Camassa-Holm equation and gave a ansätz for demonstrating the existence of a new class of solutions. In [19], Tian and Song derive some new exact peaked solitary wave solutions of the generalized CamassaHolm equation and two types of new exact traveling wave solutions of the generalized weakly dissipative CamassaHolm equations. In this paper we give a simple method for applying ordinary differential equation to solve the generalized Camassa-Holm equation and give the improved ansätz. The numerical simulation examples demonstrate that our methods are applicable.

\section{Simplification of the Nonlinear Generalized Camassa-Holm Equation}

Consider the following nonlinear generalized CamassaHolm equation:

$$
u_{t}+2 k u_{x}-u_{x x t}+a u^{m} u_{x}-2 u_{x} u_{x x}+u u_{x x x}=0 .
$$

With the velocity constant $c$, we seek the traveling wave solution of the form $u(x, t)=v(\xi)$ of (2) where $\xi=x-c t$. Substituting $u(x, t)=v(\xi)$ into (2), we have

$$
-c \frac{\mathrm{d} v}{\mathrm{~d} \xi}+2 k \frac{\mathrm{d} v}{\mathrm{~d} \xi}+c \frac{\mathrm{d}^{3} v}{\mathrm{~d} \xi^{3}}+a v^{m} \frac{\mathrm{d} v}{\mathrm{~d} \xi}-2 \frac{\mathrm{d} v}{\mathrm{~d} \xi} \frac{\mathrm{d}^{2} v}{\mathrm{~d} \xi^{2}}-v \frac{\mathrm{d}^{3} v}{\mathrm{~d} \xi^{3}}=0
$$


Integrating both sides of (3), we obtain

$$
(v-c) \frac{\mathrm{d}^{2} v}{\mathrm{~d} \xi^{2}}+\frac{1}{2}\left(\frac{\mathrm{d} v}{\mathrm{~d} \xi}\right)^{2}=\frac{a}{m+1} v^{m+1}+(2 k-c) v+b,
$$

where $b$ is an arbitrary constant. Let $\mathrm{d} v / \mathrm{d} \xi=w$. Then $\mathrm{d}^{2} v / \mathrm{d} \xi^{2}=w(\mathrm{~d} w / \mathrm{d} v)$. Substituting this into (4) yields

$$
\frac{\mathrm{d} w}{\mathrm{~d} v}+\frac{1}{2(v-c)} w=\frac{(a /(m+1)) v^{m+1}+(2 k-c) v+b}{v-c} w^{-1} .
$$

Solving (4) leads to

$$
w^{2}=(v-c)^{-1}\left\{d+\int 2\left[\frac{a}{m+1} v^{m+1}+(2 k-c) v+b\right] \mathrm{d} v\right\},
$$

where $d$ is an arbitrary constant. Therefore

$$
\begin{aligned}
& \left(\frac{\mathrm{d} v}{\mathrm{~d} \xi}\right)^{2} \\
& =(v-c)^{-1} \\
& \quad \times\left\{d+\left[\frac{2 a}{(m+1)(m+2)} v^{m+2}+(2 k-c) v^{2}+b v\right]\right\} .
\end{aligned}
$$

From $(\mathrm{d} \xi)^{2}=(\mathrm{d}|\xi|)^{2}$, we have

$$
\begin{aligned}
& \left(\frac{\mathrm{d} v}{\mathrm{~d}|\xi|}\right)^{2} \\
& =(v-c)^{-1} \\
& \quad \times\left\{d+\left[\frac{2 a}{(m+1)(m+2)} v^{m+2}+(2 k-c) v^{2}+b v\right]\right\} .
\end{aligned}
$$

Therefore

$$
\begin{aligned}
\int \sqrt{\frac{v-c}{d+\left[(2 a /((m+1)(m+2))) v^{m+2}+(2 k-c) v^{2}+b v\right]}} d v \\
=\left\{\begin{array}{l}
h+\xi, \\
h-\xi, \\
h+|\xi|, \\
h-|\xi|,
\end{array}\right.
\end{aligned}
$$

where $h$ is an arbitrary constant.

When $m=1$, (9) is the case of the Camassa-Holm equation. We take $2 k \neq c, a c-3 c+2 k=0, d=0$, and $b=0$, then

$$
\sqrt{\frac{3}{a}} \ln \frac{c}{v}=\left\{\begin{array}{l}
h+\xi, \\
h-\xi \\
h+|\xi| \\
h-|\xi|
\end{array}\right.
$$

So

$$
v=\left\{\begin{array}{l}
c \mathrm{e}^{\sqrt{(a / 3)}(h+\xi)}, \\
c \mathrm{e}^{\sqrt{(a / 3)}(h-\xi)}, \\
c \mathrm{e}^{\sqrt{(a / 3)}(h+|\xi|)} \\
c \mathrm{e}^{\sqrt{(a / 3)}(h-|\xi|)}
\end{array}\right.
$$

It can be checked that $u(x, t)=c \mathrm{e}^{h+\sqrt{(a / 3)}(x-c t)}, u(x, t)=$ $c \mathrm{e}^{h-\sqrt{(a / 3)}(x-c t)}$, and $u(x, t)=c \mathrm{e}^{h+\sqrt{(a / 3)}|x-c t|}$ are infinitely great solutions of the Camassa-Holm equation without asymptotic behavior [20]. Only

$$
u(x, t)=c \mathrm{e}^{h-\sqrt{(a / 3)}|x-c t|}
$$

are the solitary wave solutions of the Camassa-Holm equation.

When $m=2$, we take $2 k<c, a c^{2}-6 c+12 k=0, d=0$, and $b=0$. In the similar way in the case $m=1$, we only choose

$$
\sqrt{\frac{6}{a}} \int \frac{1}{v \sqrt{v+c}} \mathrm{~d} \varphi=h-|\xi|
$$

then

$$
-\sqrt{\frac{24}{a c}} \operatorname{atanh} \sqrt{\frac{v+c}{c}}=h-|\xi| \text {. }
$$

From (14), we have

$$
v=c \tanh ^{2}\left[\sqrt{\frac{a c}{24}}(|\xi|-h)\right]-c
$$

Therefore we have the solitary wave solutions of the generalized Camassa-Holm equation as

$$
u(x, t)=c \tanh ^{2}\left[\sqrt{\frac{a c}{24}}(|x-c t|-h)\right]-c .
$$

When $m=3$, we take $2 k \neq c, a c^{3}-10 c+20 k=0, d=0$, and $b=0$ then

$$
\sqrt{\frac{10}{a}} \int \frac{1}{\sqrt{v^{2}+c v+c^{2}}} \mathrm{~d} v=h-|\xi|
$$

Then

$$
-\sqrt{\frac{10}{a c^{2}}} \operatorname{atanh} \frac{c(v+2 c)}{|c| \sqrt{v^{2}+c v+c^{2}}}=h-|\xi| .
$$


Solving (18), we obtain

$$
\begin{gathered}
v=\left(-2 c\left\{1-\tanh ^{2}\left[\sqrt{\frac{a c^{2}}{10}}(|\xi|-h)\right]\right\}\right. \\
\pm 2|c|\left(3\left\{1-\tanh ^{2}\left[\sqrt{\frac{a c^{2}}{10}}(|\xi|-h)\right]\right\}\right. \\
\left.\left.\times \tanh ^{2}\left[\sqrt{\frac{a c^{2}}{10}}(|\xi|-h)\right]\right)^{1 / 2}\right) \\
\times\left(1-4 \tanh ^{2}\left[\sqrt{\frac{a c^{2}}{10}}(|\xi|-h)\right]\right)^{-1} .
\end{gathered}
$$

Therefore

$$
\begin{aligned}
u(x, t)= & \left(-2 c\left[1-\tanh ^{2} f(x-c t)\right]\right. \\
& \left.+2|c| \sqrt{3\left[1-\tanh ^{2} f(x-c t)\right] \tanh ^{2} f(x-c t)}\right) \\
& \times\left(1-4 \tanh ^{2} f(x-c t)\right)^{-1}
\end{aligned}
$$

or

$$
\begin{aligned}
u(x, t)= & \left(-2 c\left[1-\tanh ^{2} f(x-c t)\right]\right. \\
& \left.-2|c| \sqrt{3\left[1-\tanh ^{2} f(x-c t)\right] \tanh ^{2} f(x-c t)}\right) \\
\times & \left(1-4 \tanh ^{2} f(x-c t)\right)^{-1},
\end{aligned}
$$

where $f(x-c t)=\sqrt{\left(a c^{2} / 10\right)}(|x-c t|-h)$.

\section{Ansätz for the Generalized Camassa-Holm Equation}

From [18], we have the following ordinary differential equation in $F$ :

$$
\begin{aligned}
2 F^{3} \frac{\mathrm{d}^{2} F}{\mathrm{~d} \xi^{2}}+4 F^{2}\left(\frac{\mathrm{d} F}{\mathrm{~d} \xi}\right)^{2}= & \frac{a}{m+1} \sum_{k=0}^{m+1}\left(\begin{array}{c}
m+1 \\
k
\end{array}\right) F^{2 k} c^{m+1-k} \\
& +(2 k-c) F^{2}+2 k c-c^{2}+b
\end{aligned}
$$

where $F=\sqrt{v-c}$.
Let $\mathrm{d} F / \mathrm{d} \xi=G$. Then $\mathrm{d}^{2} F / \mathrm{d} \xi^{2}=G(\mathrm{~d} G / \mathrm{d} F)$. Substituting this into (22) gives the following first order Bernoulli's ordinary differential equation:

$$
\begin{aligned}
\frac{\mathrm{d} G}{\mathrm{~d} F}+\frac{2}{F} G=( & \left(\frac{a}{m+1} \sum_{k=0}^{m+1}\left(\begin{array}{c}
m+1 \\
k
\end{array}\right) F^{2 k} c^{m+1-k}\right. \\
& \left.+(2 k-c) F^{2}+2 k c-c^{2}+b\right) \\
& \left.\times\left(2 F^{3}\right)^{-1}\right) G^{-1} .
\end{aligned}
$$

Solving (23) leads to

$$
\begin{aligned}
G^{2}=F^{-4}\left\{g+\int\left[\frac{a}{m+1} \sum_{k=0}^{m+1}\left(\begin{array}{c}
m+1 \\
k
\end{array}\right) F^{2 k} c^{m+1-k}\right.\right. \\
\left.\left.+(2 k-c) F^{2}+2 k c-c^{2}+b\right] F \mathrm{~d} F\right\} .
\end{aligned}
$$

Therefore

$$
\begin{aligned}
\left(F \frac{\mathrm{d} F}{\mathrm{~d} \xi}\right)^{2}=g F^{-2}+ & {\left[\frac{a}{m+1} \sum_{k=0}^{m+1} \frac{1}{2 k+2}\left(\begin{array}{c}
m+1 \\
k
\end{array}\right) F^{2 k} c^{m+1-k}\right.} \\
& \left.+\frac{1}{4}(2 k-c) F^{2}+\frac{1}{2}\left(2 k c-c^{2}+b\right)\right],
\end{aligned}
$$

where $g$ is an arbitrary constant. We observe that $F(\mathrm{~d} F / \mathrm{d} \xi)$ hardly becomes a polynomial in $F$ unless in the particular cases. So this is a new ansätz compared with the ansätz in [18]. Similarly, we have

$$
\begin{aligned}
\left(F \frac{\mathrm{d} F}{\mathrm{~d}|\xi|}\right)^{2}=g F^{-2}+ & {\left[\frac{a}{m+1} \sum_{k=0}^{m+1} \frac{1}{2 k+2}\left(\begin{array}{c}
m+1 \\
k
\end{array}\right) F^{2 k} c^{m+1-k}\right.} \\
& \left.+\frac{1}{4}(2 k-c) F^{2}+\frac{1}{2}\left(2 k c-c^{2}+b\right)\right] .
\end{aligned}
$$

Seemingly, (26) is more difficult than (8). On the contrary, (26) is much easier to be solved than (8). This can be seen in the following.

When $m=1$, let $g=0$. For $F^{2}=\theta$, we have

$$
\begin{aligned}
\int\left(\frac{a}{3} \theta^{2}+\right. & \frac{1}{4}(2 k-c+4 a c) \theta \\
& \left.+\frac{1}{2}\left(2 k c-c^{2}+2 a c^{2}+b\right)\right)^{-1 / 2} \mathrm{~d} \theta=r-|\xi|
\end{aligned}
$$


where $r$ is an arbitrary constant. So

$$
\begin{aligned}
& \sqrt{\frac{3}{a}} \ln [ {\left[\theta+\frac{3(2 k-c+4 a c)}{8 a}\right) } \\
&\left.+\sqrt{\theta^{2}+\frac{3(2 k-c+4 a c)}{4 a} \theta+\frac{3\left(2 k c-c^{2}+2 a c^{2}+b\right)}{2 a}}\right] \\
& \quad=r-|\xi| .
\end{aligned}
$$

Solving (28), we have that when $3\left(2 k c-c^{2}+2 a c^{2}+b\right) /(2 a)-$ $9(2 k-c+4 a c)^{2} /\left(16 a^{2}\right)>0$,

$$
\begin{aligned}
\theta= & \sqrt{\frac{3\left(2 k c-c^{2}+2 a c^{2}+b\right)}{2 a}-\frac{9(2 k-c+4 a c)^{2}}{16 a^{2}}} \\
& \times \sinh \left[\sqrt{\frac{3}{a}}(\bar{r}-|\xi|)\right]-\frac{3(2 k-c+4 a c)}{8 a}
\end{aligned}
$$

when $3\left(2 k c-c^{2}+2 a c^{2}+b\right) /(2 a)-9(2 k-c+4 a c)^{2} /\left(16 a^{2}\right)<0$,

$$
\begin{aligned}
\theta= & \sqrt{\frac{3\left(2 k c-c^{2}+2 a c^{2}+b\right)}{2 a}-\frac{9(2 k-c+4 a c)^{2}}{16 a^{2}}} \\
& \times \cosh \left[\sqrt{\frac{3}{a}}(\bar{r}-|\xi|)\right]-\frac{3(2 k-c+4 a c)}{8 a} .
\end{aligned}
$$

Equations (29) and (30) have no asymptotic behavior. When $3\left(2 k c-c^{2}+2 a c^{2}+b\right) /(2 a)=9(2 k-c+4 a c)^{2} /\left(16 a^{2}\right)$, we obtain

$$
\theta=\frac{1}{2} \mathrm{e}^{\sqrt{(a / 3)}(r-|\xi|)}-\frac{3(2 k-c+4 a c)}{16 a} .
$$

So

$$
u(x, t)=\frac{1}{2} \mathrm{e}^{\sqrt{(a / 3)}(r-|x-c t|)}-\frac{6 k-3 c-4 a c}{16 a} .
$$

These solitary solutions have the first derivative discontinuity at the wave peak.

When $m=2$, let $g=0$. Consider the following:

$$
\begin{aligned}
\int\left(\frac{a}{6} \theta^{3}+\frac{2 a c}{3} \theta^{2}\right. & +\frac{1}{4}\left(2 k-c+4 a c^{2}\right) \theta \\
& \left.+\frac{1}{2}\left(2 k-c^{2}+\frac{4 a c^{3}}{3}+b\right)\right)^{-1 / 2} \mathrm{~d} \theta=r-|\xi|
\end{aligned}
$$

Take $2 k-c+4 a c^{2}=0$ and $2 k-c^{2}+4 a c^{3} / 3+b=0$, then

$$
-\sqrt{\frac{6}{a c}} \operatorname{atanh} \sqrt{\frac{\theta+4 c}{4 c}}=r-|\xi| .
$$

From (34), we have

$$
\theta=4 c \tanh ^{2}\left[\sqrt{\frac{a c}{6}}(|\xi|-r)\right]-4 c
$$

Therefore, we have the solitary wave solutions of the generalized Camassa-Holm equation as

$$
u(x, t)=4 c \tanh ^{2}\left[\sqrt{\frac{a c}{6}}(|x-c t|-r)\right]-3 c .
$$

Take $2 k-c+4 a c^{2}=8 a c^{2} / 3$ and $2 k-c^{2}+4 a c^{3} / 3+b=0$, then

$$
\sqrt{\frac{12}{a c}} \operatorname{atan} \sqrt{\frac{\theta}{2 c}}=r-|\xi| \text {. }
$$

Similarly, we have

$$
u(x, t)=2 c \tan ^{2}\left[\sqrt{\frac{a c}{12}}(r-|x-c t|)\right]+c .
$$

This result is the same as (28) in [18]. But it is easily to obtain.

When $m=3$, let $g=0$. Consider the following:

$$
\begin{aligned}
\int\left(\frac{a}{10} \theta^{4}+\frac{a c}{2} \theta^{3}+a c^{2} \theta^{2}+\frac{1}{4}\left(2 k-c+4 a c^{3}\right) \theta\right. \\
\left.+\frac{1}{2}\left(2 k-c^{2}+a c^{4}+b\right)\right)^{-1 / 2} \mathrm{~d} \theta=r-|\xi| .
\end{aligned}
$$

From (39) and $a, c>0$, we have seen that $F(\mathrm{~d} F / \mathrm{d} \xi)$ is not a polynomial in $F$ (compared with Equation (35) in [18]). Take $2 k-c+4 a c^{3}=0$ and $2 k-c^{2}+a c^{4}+b=0$, then

$$
-\sqrt{\frac{1}{a c^{2}}} \operatorname{atanh} \frac{\sqrt{10}\left(c \theta+4 c^{2}\right)}{4|c| \sqrt{\theta^{2}+5 c \theta+10 c^{2}}}=r-|\xi| .
$$

Solving (40), we obtain

$$
\begin{aligned}
\theta= & \left(-20 c\left[1-\tanh ^{2} p(\xi)\right]\right. \\
& \left. \pm 4|c| \sqrt{15\left[1-\tanh ^{2} p(\xi)\right] \tanh ^{2} p(\xi)}\right) \\
& \times\left(5-8 \tanh ^{2} p(\xi)\right)^{-1},
\end{aligned}
$$

where $p(\xi)=\sqrt{a}|c|(|\xi|-r)$. Therefore

$$
\begin{aligned}
u(x, t)=(( & -20 c\left[1-\tanh ^{2} p(x-c t)\right]+4|c| \\
& \left.\times \sqrt{15\left[1-\tanh ^{2} p(x-c t)\right] \tanh ^{2} p(x-c t)}\right) \\
& \left.\times\left(5-8 \tanh ^{2} p(x-c t)\right)^{-1}\right)+c,
\end{aligned}
$$

or

$$
\begin{aligned}
u(x, t)=(( & -20 c\left[1-\tanh ^{2} p(x-c t)\right]-4|c| \\
& \left.\times \sqrt{15\left[1-\tanh ^{2} p(x-c t)\right] \tanh ^{2} p(x-c t)}\right) \\
& \left.\times\left(5-8 \tanh ^{2} p(x-c t)\right)^{-1}\right)+c,
\end{aligned}
$$

where $p(x-c t)=\sqrt{a}|c|(|x-c t|-r)$. 


\section{Numerical Simulation Examples}

Example 1. In (12), take $c=1, h=0$, and $a=3$. Then the solitary wave solution is $u(x, t)=e^{-|x-t|}$.

We want to show figures with peakon feature. But to save space, omitting figures we only give MATLAB program: “ $x=$ $-10: 0.001: 10 ; u=\exp (-\operatorname{abs}(x)) ; \operatorname{plot}(x, u)$ ” for $t=0$ and "for $n=1: 101 x(n)=(n-1) / 5-10 ; t(n)=x(n)$; end $[x(n), t(n)]=\operatorname{meshgrid}(x(n), t(n))$; for $n=1: 101$ for $m=$ $1: 101 u(n, m)=\exp (-\operatorname{abs}(x(n)-t(m)))$; end end mesh $(u) "$ for the 3-dimensional case.

Example 2. In (16), take $c=1, h=0$, and $a=24$. Then solitary wave solution is $u(x, t)=\tanh ^{2}(|x-t|-1)-1$.

The figures with peakon feature will be constructed by “ $x=-10: 0.001: 10 ; u=(\tanh (\operatorname{abs}(x)-1)) .^{\wedge} 2-1 ; \operatorname{plot}(x, u) "$ for $t=0$ and "for $n=1: 101 x(n)=(n-1) / 5-10 ; t(n)=x(n)$; end $[x(n), t(n)]=\operatorname{meshgrid}(x(n), t(n))$; for $n=1: 101$ for $m=1: 101 u(n, m)=(\tanh (\operatorname{abs}(x(n)-t(m))-1)) \cdot{ }^{\wedge} 2-1$; end end $\operatorname{mesh}(u)$ " for the 3-dimensional case.

Example 3. In (20), take $c=1, h=1$, and $a=10$. Then symmetrically solitary wave solution is

$$
\begin{aligned}
& u(x, t) \\
& =\left\{-2\left[1-\tanh ^{2}(|x-t|-1)\right]\right. \\
& \left.+2 \sqrt{3\left[1-\tanh ^{2}(|x-t|-1)\right] \tanh ^{2}(|x-t|-1)}\right\} \\
& \times\left(1-4 \tanh ^{2}(|x-t|-1)\right)^{-1}
\end{aligned}
$$

For $t=0$, when $(a=-2, b=2),(a=2.31, b=2.32)$, and $(a=2.32, b=5)$, respectively, the figures are with peakon feature. $x=\mathrm{a}: 0.0001: \mathrm{b} ; y=\left(-2 . *\left(1-\tanh (\operatorname{abs}(x)-1) .{ }^{\wedge} 2\right)+\right.$ 2. $* \operatorname{sqrt}(3 . *(1-\tanh (\operatorname{abs}(x)-1) . \wedge 2) . * \tanh (\operatorname{abs}(x)))) . /(1-4 . *$ $\left.\left(1-\tanh (\operatorname{abs}(x)-1) .^{\wedge} 2\right)\right) ; \operatorname{plot}(x, y)$. Replace $x$ by $x(n)-t(m)$ and the others are similar to Example 2 program for the 3 dimensional case.

\section{Conclusions}

In this paper, we make simplification of the nonlinear generalized Camassa-Holm equation and give the improved ansatz for the generalized Camassa-Holm equation. The three numerical simulation examples with mATLAB programs demonstrate solitary wave solutions with peakon feature which show our method applicable. This method may be applied to many other nonlinear equations.

\section{Acknowledgments}

This work was supported partially by National Natural Science Foundation of China (Grant nos. 10871056 and 10971150) and by Science Research Foundation in Harbin Institute of Technology (Grant no. HITC200708).

\section{References}

[1] R. Camassa and D. D. Holm, "An integrable shallow water equation with peaked solitons," Physical Review Letters, vol. 71, no. 11, pp. 1661-1664, 1993.

[2] Y. Long, Z. Li, and W. Rui, "New travelling wave solutions for a nonlinearly dispersive wave equation of Camassa-Holm equation type," Applied Mathematics and Computation, vol. 217, no. 4, pp. 1315-1320, 2010.

[3] Y. Xu and L. Tian, "Attractor for a coupled nonhomogeneous Camassa-Holm equation," International Journal of Nonlinear Science, vol. 9, no. 1, pp. 118-122, 2010.

[4] M. Song and Z. R. Liu, "Traveling wave solutions for the generalized Zakharov equations," Mathematical Problems in Engineering, vol. 2012, Article ID 747295, 14 pages, 2012.

[5] S. Tang, Y. Xiao, and Z. Wang, "Travelling wave solutions for a class of nonlinear fourth order variant of a generalized Camassa-Holm equation," Applied Mathematics and Computation, vol. 210, no. 1, pp. 39-47, 2009.

[6] B. He, W. Rui, C. Chen, and S. Li, "Exact travelling wave solutions of a generalized Camassa-Holm equation using the integral bifurcation method," Applied Mathematics and Computation, vol. 206, no. 1, pp. 141-149, 2008.

[7] A.-M. Wazwaz, "New solitary wave solutions to the modified forms of Degasperis-Procesi and Camassa-Holm equations," Applied Mathematics and Computation, vol. 186, no. 1, pp. 130141, 2007.

[8] A.-M. Wazwaz, "Peakons, kinks, compactons and solitary patterns solutions for a family of Camassa-Holm equations by using new hyperbolic schemes," Applied Mathematics and Computation, vol. 182, no. 1, pp. 412-424, 2006.

[9] S. Chen, C. Foias, D. D. Holm, E. Olson, E. S. Titi, and S. Wynne, "Camassa-Holm equations as a closure model for turbulent channel and pipe flow," Physical Review Letters, vol. 81, no. 24, pp. 5338-5341, 1998.

[10] F. Cooper and H. Shepard, "Solitons in the Camassa-Holm shallow water equation," Physics Letters A, vol. 194, no. 4, pp. 246-250, 1994

[11] M. Fisher and J. Schiff, "The Camassa Holm equation: conserved quantities and the initial value problem," Physics Letters A, vol. 259, no. 5, pp. 371-376, 1999.

[12] Z. Liu, T. Qian, and M. Tang, "Peakons of the Camassa-Holm equation," Applied Mathematical Modelling, vol. 26, pp. 473480, 2002.

[13] Z. Liu and T. Qian, "Peakons and their bifurcation in a generalized Camassa-Holm equation," International Journal of Bifurcation and Chaos in Applied Sciences and Engineering, vol. 11, no. 3, pp. 781-792, 2001.

[14] T. Qian and M. Tang, "Peakons and periodic cusp waves in a generalized Camassa-Holm equation," Chaos, Solitons and Fractals, vol. 12, no. 7, pp. 1347-1360, 2001.

[15] R. A. Kraenkel, M. Senthilvelan, and A. I. Zenchuk, "On the integrable perturbations of the Camassa-Holm equation," Journal of Mathematical Physics, vol. 41, no. 5, pp. 3160-3169, 2000.

[16] L. Tian and J. Yin, "New compacton solutions and solitary wave solutions of fully nonlinear generalized Camassa-Holm equations," Chaos, Solitons and Fractals, vol. 20, no. 2, pp. 289299, 2004. 
[17] Z. Liu, T. Jiang, P. Qin, and Q. Xu, "Trigonometric function periodic wave solutions and their limit forms for the KdVlike and the PC-like equations," Mathematical Problems in Engineering, vol. 2011, Article ID 810217, 23 pages, 2011.

[18] S. A. Khuri, "New ansätz for obtaining wave solutions of the generalized Camassa-Holm equation," Chaos, Solitons \& Fractals, vol. 25, no. 3, pp. 705-710, 2005.

[19] L. Tian and X. Song, "New peaked solitary wave solutions of the generalized Camassa-Holm equation," Chaos, Solitons and Fractals, vol. 19, no. 3, pp. 621-637, 2004.

[20] R. Camassa and A. I. Zenchuk, "On the initial value problem for a completely integrable shallow water wave equation," Physics Letters A, vol. 281, no. 1, pp. 26-33, 2001. 


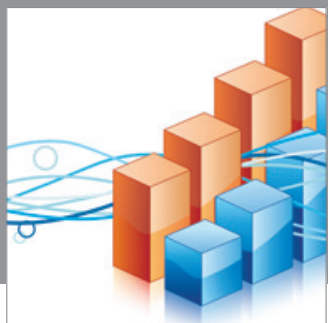

Advances in

Operations Research

mansans

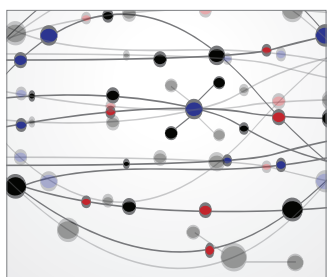

The Scientific World Journal
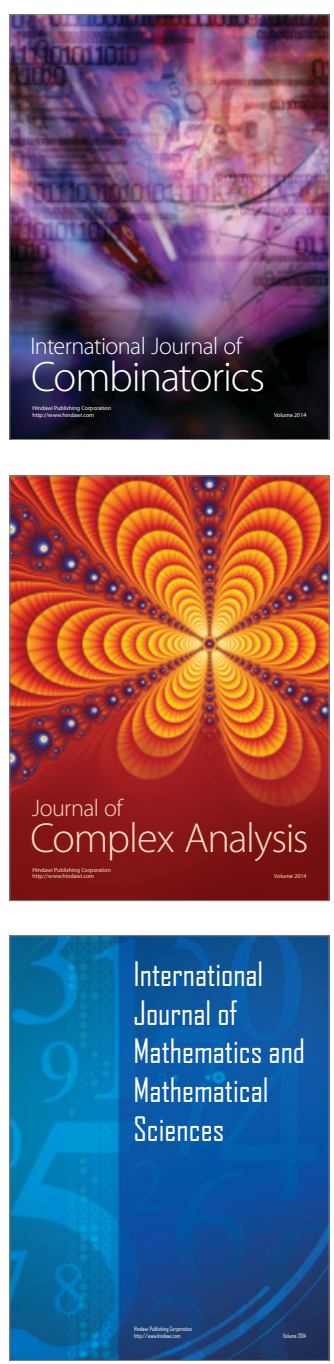
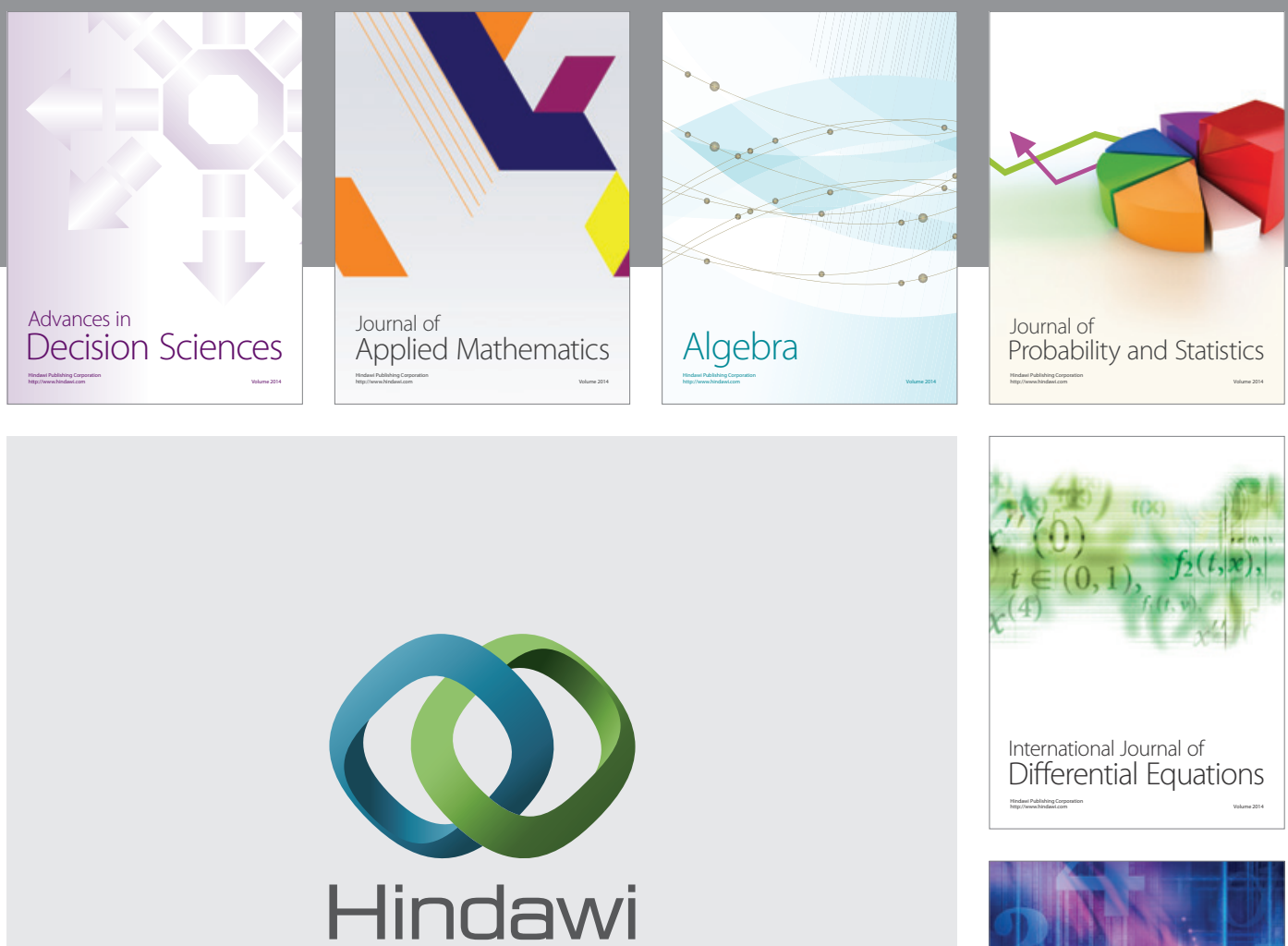

Submit your manuscripts at http://www.hindawi.com
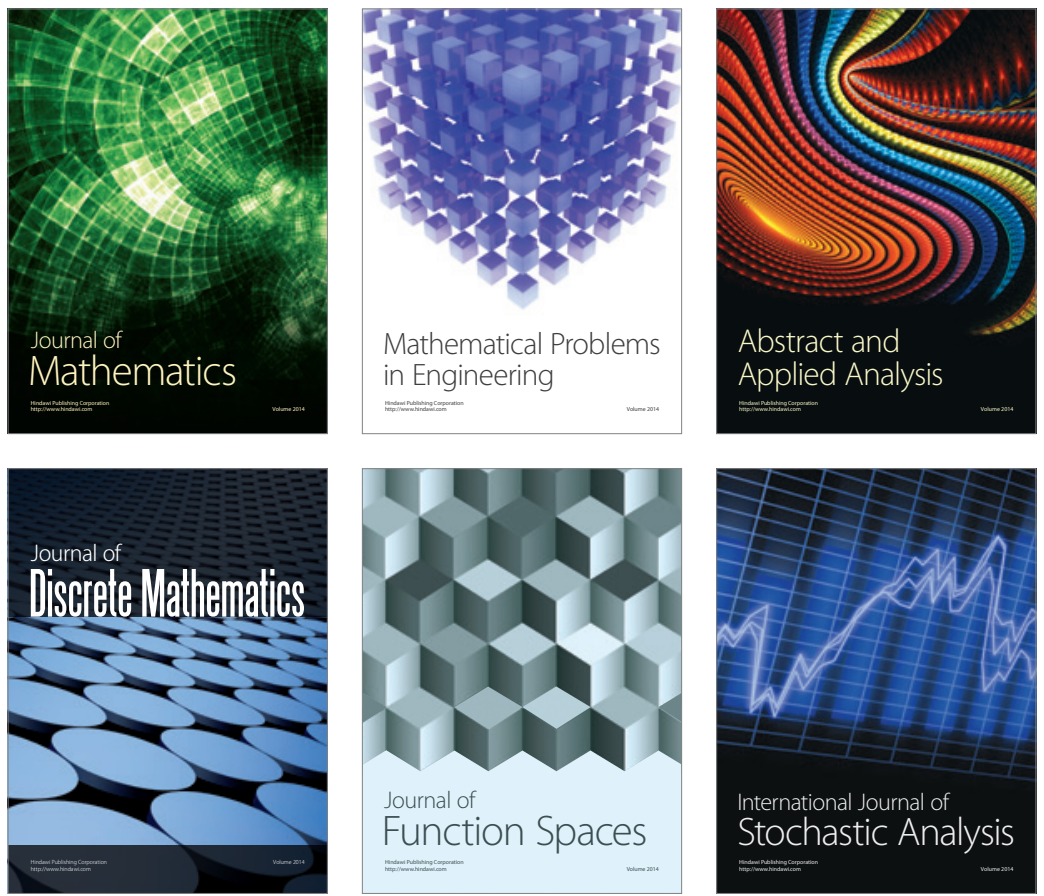

Journal of

Function Spaces

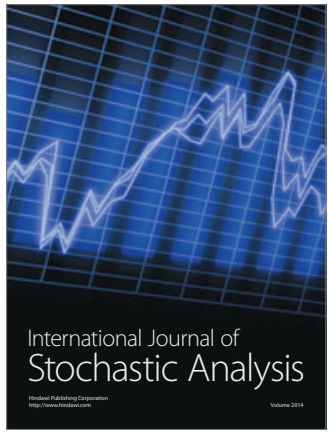

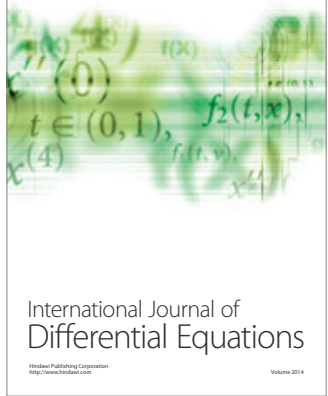
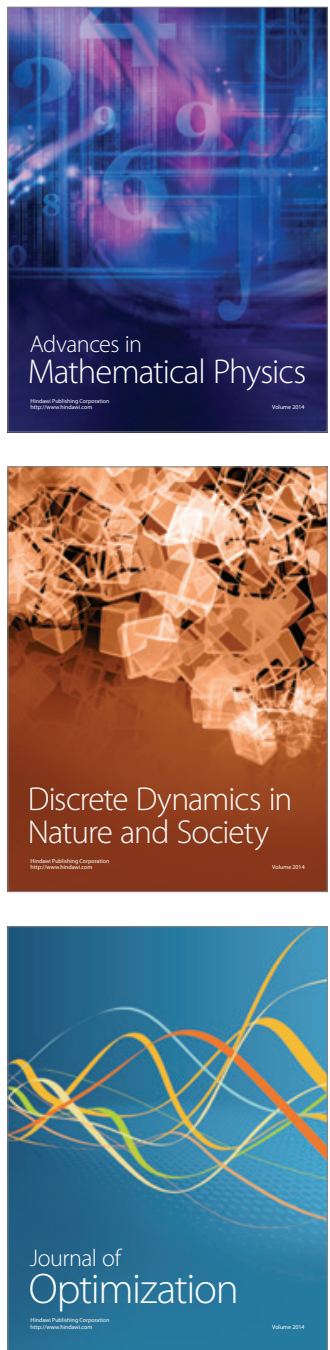